\title{
The long and the short of it: are shorter periods of hospitalisation beneficial?
}

\author{
Delphine Capdevielle and Karen Ritchie
}

\section{Summary}

The politics of shortening hospital stays for people with psychosis has been questioned by a number of studies. Hospital practice in the meantime remains highly variable and the research evidence increasingly difficult to interpret given different conceptualisations of what constitutes effective treatment. In the absence of clearer guidelines from researchers in this area, decisions about duration of hospitalisation risk being driven by economic rather than clinical considerations.

\section{Declaration of interest}

None.
Delphine Capdevielle (pictured) is a psychiatrist working in the University Department of Adult Psychiatry at the $\mathrm{CHU}$ de Montpellier and with the French National Institute of Medical Research U-888. Karen Ritchie is a neuropsychologist and epidemiologist and is a research director with the French National Institute of Medical Research.

A lasting consequence of socio-environmentalist theories of psychosis emanating from the 1960s has been the belief that patients with psychosis benefit from a rapid return to the community, thus minimising social isolation and the adverse effects of adopting psychiatric communities as reference groups. Studies of patients returning to the community compared with those remaining in institutions show not only better quality of life and larger friendship networks, but also reductions in dependence on pharmacotherapies and lower mortality rates. ${ }^{1}$ Enthusiasm for the shortening of hospital stays in the interest of more rapid community reintegration has undoubtedly also been echoed by health economists; the average cost of hospital stays for people with psychotic disorders being estimated between $€ 2830.29$ and $€ 3624.95$ of which only $3.4 \%$ is related to diagnosis and $2.4 \%$ to actual treatment. ${ }^{2}$ In almost all Western countries the past 40 years has seen a general transition towards a policy of reduced periods of hospitalisation of patients with psychosis and a reduced number of hospital beds.

On the other hand there has been some unsettling evidence to suggest that shortening hospital stays may not be a general panacea. Munk-Jorgensen et al reported that deinstitutionalisation policies in Denmark had led to premature discharge and subsequently a $100 \%$ increase in suicide, a doubling of criminal acts committed by patients with psychoses and increases of $80-100 \%$ in acute admission rates. ${ }^{3}$ In a large follow-up study of patients with psychoses, Desai et al found that hospital stays of less than 14 days were significantly associated with increased suicide risk. ${ }^{4}$ Other studies have shown a clear relationship between reductions in duration of stay and increases in readmission rates. ${ }^{5}$ These observations have led to speculation that early discharge leads to inadequate treatment; an argument that has been further fuelled in France and the UK by highly publicised homicides committed by psychiatric in-patients. Others have argued that negative outcomes are attributable to the fact that patients with psychosis, who have drug and alcohol problems, and thus a poorer prognosis, are more likely to seek premature discharge and to discontinue treatment. ${ }^{6}$ A Cochrane meta-analysis conducted in 2000 concluded that shorter periods of hospitalisation did not increase the risk of readmission when the hospitalisation is programmed and planned in advance as a short-term admission. ${ }^{7}$

\section{Short- $v$. long-term hospitalisation}

An interesting comparison between three contrasting mental healthcare systems in Holland, Italy and Australia ${ }^{8}$ concluded, not surprisingly, that hospital stays are shortest where community care is more developed, although long-term hospitalisation will always be required for a small number of patients who are severely ill. The general conclusion was that shorter stays work best if and only if there is high-quality community care that comes into play immediately on discharge. The central issue appears to be that the beneficial effects of short stays are modulated by conditions of discharge. That is, in the absence of a planned discharge policy, patients appear to be better off staying longer in order that a structured rehabilitation plan may be put in place. This is clearly a very high-cost strategy.

Thus, unlike most other causes of hospitalisation, duration of in-patient treatment for psychosis is not only determined by treatment considerations and rate of recovery but also by factors relating to the personal circumstances of the patient and organisation of the health system. It has been estimated that only $50 \%$ of hospitalisations over 30 days are medically justified. ${ }^{9}$ Of the remainder, $40 \%$ are due to social problems and $10 \%$ are 'medically unacceptable'.

The evidence presently available suggests that short-term stays may be preferable for certain patients, but that these stays should be planned with structured discharge programmes. That is all very well, but which patients are best suited to short-term stays? What is 'short term' (studies cite periods varying from 2 weeks to a year)? Which rehabilitation programmes should be employed? Should rehabilitation planning be a reason for extending hospitalisation? Does rehospitalisation constitute a failure of therapy or effective prevention? Perhaps the most important point has been made by Szmukler \& Holloway; ${ }^{10}$ despite the extensive literature on hospital care, we still cannot define effective in-patient treatment.

\section{Future considerations}

While few patients or clinicians would contest today that a return to normal community life is preferable to institutionalisation, the adoption of shorter hospital stays was not an evidence-based policy and no adequate evaluation study was ever put in place to predict the social, clinical and economic consequences of this widespread practice. Although duration of hospitalisation is a factor likely to have an enormous impact on the course of the 
illness, quality of life, long-term social and professional reintegration, not to mention the costs to the health sector, we are far from having clear directives on best practice, or even for that matter consensus on what constitutes a short stay. Hospital policy in the meantime remains highly variable, determined principally by available resources rather than evidence-based guidelines. Moreover, as early evidence suggests that psychogenetic screening for positive treatment response to specific antipsychotics will in the future be an important means of reducing hospitalisation time, research in this area now needs to take into account the potential impact of this innovation. As the European Community works towards the standardisation of health delivery protocols in other areas of medicine there is a clear need to reinvest in research of care for patients with psychoses in order to develop clearer best practice guidelines.

Delphine Capdevielle, MD, Karen Ritchie, PhD, French National Institute of Health and Medical Research, INSERM U-888, La Colombière Hospital, 34093 Montpellier Cedex 5, France

Correspondence: D. Capdevielle, French National Institute of Health and Medical Research, INSERM U-888, La Colombière Hospital, 34093 Montpellier Cedex 5, France. Email: delphine.capdevielle@free.fr

First received 5 Sep 2007, final revision 5 Sep 2007, accepted 7 Dec 2007

\section{Acknowledgement}

We thank Professor Graham Thornicroft for his useful comments on this paper.

\section{References}

1 Ryu Y, Mizuno M, Sakuma K, Munakata S, Takebayashi T, Murakami M, Falloon IR, Kashima H. Deinstitutionalization of long-stay patients with schizophrenia: the 2-year social and clinical outcome of a comprehensive intervention program in Japan. Aust N Z J Psychiatry 2006; 40: 462-70.

2 Peiró S, Gómez G, Navarro M, Guadarrama I, Rejas J; on behalf of the Psychosp Group. Length of stay and antipsychotic treatment costs of patients with acute psychosis admitted to hospital in Spain. Description and associated factors. The Psychosp study. Soc Psychiatry Psychiatr Epidemiol 2004; 39: 507-13.

3 Munk-Jorgensen P. Has deinstitutionalization gone too far? Eur Arch Psychiatry Clin Neurosci 1999; 249: 136-43.

4 Desai RA, Dausey DJ, Rosenheck RA. Mental health service delivery and suicide risk: the role of individual patient and facility factors. Am J Psychiatry 2005; 162: 311-18.

5 Lin HC, Tian WH, Chen CS, Liu TC, Tsai SY, Lee HC. The association between readmission rates and length of stay for schizophrenia: a 3-year populationbased study. Schizophr Res 2006; 83: 211-14.

6 Ries RK, Russo J, Wingerson D, Snowden M, Comtois KA, Srebnik D, Roy-Byrne P. Shorter hospital stays and more rapid improvement among patients with schizophrenia and substance disorders. Psychiatr Serv 2000; 51: 210-15.

7 Johnstone $\mathrm{P}$, Zolese $\mathrm{G}$. Length of hospitalisation for people with severe mental illness. Cochrane Database Syst Rev 2000; 2: CD000384.

8 Sytema S, Burgess P, Tansella M. Does community care decrease length of stay and risk of rehospitalization in new patients with schizophrenia disorders? A comparative case register study in Groningen, The Netherlands; Victoria, Australia; and South-Verona, Italy. Schizophr Bull 2002; 28: 273-81.

9 Hallak JE, Crippa JA, Vansan G, Zuardi AW. Diagnostic profile of inpatients as a determinant of length of stay in a general hospital psychiatric unit. Braz $J$ Med Biol Res 2003; 36: 1233-40.

10 Szmukler G, Holloway F. Reform of the Mental Health Act. Health or safety? Br J Psychiatry 2000; 177: 196-200 